\title{
FORMULATION AND ANALYSIS OF A FUNCTIONAL EQUATION DESCRIBING A MOVING ONE-DIMENSIONAL ELASTIC PHASE BOUNDARY*
}

\author{
BY \\ THOMAS J. PENCE \\ University of Wisconsin**
}

\begin{abstract}
Certain elastic solids when subjected to sufficiently high loads abruptly change their mechanical properties and yet continue to respond elastically to further loading. In one dimension such mechanically induced elastic phase transitions may be due to a nonmonotonic stress-strain relationship. This appears to be particularly true for certain mineral crystals, such as calcite.

This work considers a one-dimensional dynamical problem for a special material. The problem reduces to determining the location of the internal moving boundary separating distinct elastic phases. This phase boundary is similar to a gas dynamical shock wave. For the problem considered here, this phase boundary is shown to be governed by a functional equation of the form

$$
\varphi(\varphi(t))+F(\varphi(t))+t=0,
$$

for the unknown $\varphi(t)$, where $F(\cdot)$ is a known function involving the boundary conditions. The unusual equation is derived by considering the effect of acoustic waves reflecting repeatedly between the phase boundary and the external boundary. The equation is shown to possess a unique solution and can be treated asymptotically to determine the behavior of the phase boundary after short and long time periods.
\end{abstract}

1. Introduction. One of the objectives of the one-dimensional dynamical theory of displacive elastic phase transitions is to determine the motion of the internal moving boundary separating different elastic phases. An example of such motion occurs in the

\footnotetext{
* Received January 23, 1986. This work was sponsored by the United States Army under Contract No. DAAG29-80-C-0041, and is based upon work supported by the National Science Foundation under Grant No. MCS- 8210950 .

** The author's permanent address is the Department of Metallurgy, Mechanics and Materials Science, Michigan State University.
} 
mineral calcite, where a moving front can separate different crystal structures [1]. Models for such displacive phase transitions have been proposed which, in one dimension, involve a nonmonotonic stress-strain relation [2,3]. Moving phase boundaries in these models are kinematically similar to other types of shock fronts in that velocity and strain are discontinuous across them. One difference is that even at first formation, say $t=0$, the jump in strain may be large $[4,5]$. The simplest of these models lead to solutions with a high degree of nonuniqueness. Resolution of the admissibility question for such theories has led to more detailed modeling of the phase change zone, where certain effects in the simpler theories can no longer by presumed small $[6,7,8]$. The object of this study is to treat an aspect of the original kinematic theory involving sharp-fronted phase boundaries, namely, how to determine the trajectory of such a moving boundary when it is being driven forward by acoustic waves ringing between it and a loading device. In certain cases this problem is reducible to a functional equation of the form

$$
\varphi(\varphi(t))+F(\varphi(t))+t=0,
$$

where $F(\cdot)$ embodies the loading conditions, and the function to be determined, $\varphi$, is subject to some side conditions. We sketch the derivation of this equation and then show that it possesses a unique, continuously differentiable solution on $t \geqslant 0$. In general, higher derivatives at $t=0$ will not exist unless additional restrictions are imposed on the loading conditions. In addition, one of the theorems presented here furnishes an iterative scheme for solving this functional equation. An interesting feature of this scheme is that it does not require a preliminary analysis of $\varphi(t)$ on an initial interval. This contrasts with methods employed for treating a set of functional equations arising in the theory of plastic unloading waves $[9,10]$.

While uniqueness of solutions to the functional equation is proved, this equation is derived under a number of assumptions stated in the following section. As such, the analysis presented here in no way implies uniqueness of solutions to initial-boundary value problems for materials which are characterized solely by a nonmonotonic stress-strain relation.

2. Formulation of the problem. Consider a semi-infinite bar occupying $x \geqslant 0$ to which a load is being applied at the end $x=0$. Let

$$
u(x, t), \quad \varepsilon=\partial u / \partial x, \quad v=\partial u / \partial t, \quad \sigma(\varepsilon),
$$

denote, respectively, the longitudinal displacement, strain, velocity, and stress in the bar. Our essential assumptions are:

(i) The loading device initiates a change of phase at $t=0$. Specifically we consider an applied positive traction which at $t=0$ induces at $x=0$ a finite jump in strain from $\varepsilon_{\mathrm{I}}$ to $\varepsilon^{*}$ due to the material admitting multiple strain phases at the value of stress $\sigma^{*}$ (Fig. 1). Afterward the end-strain is assumed to increase smoothly. We write this boundary condition thus:

$$
\varepsilon(0, t)=h(t), \quad h(0-)=\varepsilon_{\mathrm{I}}, \quad h(0+)=\varepsilon^{*}
$$




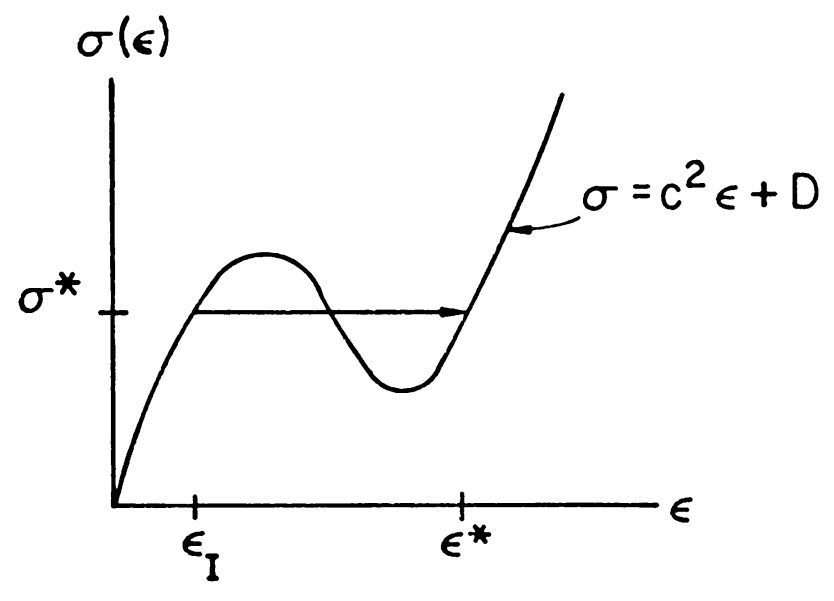

FIG. 1

where $\sigma\left(\varepsilon_{\mathrm{I}}\right)=\sigma\left(\varepsilon^{*}\right)=\sigma^{*}$. For $t>0, h(t)$ is assumed to be of unlimited smoothness with $h(t)>\varepsilon^{*}$.

(ii) The strain and velocity in the bar are initially constant at the values $\varepsilon_{\mathrm{I}}, v_{\mathrm{I}}$ before the phase boundary emerges at $t=0$.

(iii) The material in the second phase obeys a linear stress-strain law over the range of subsequent loading. Hence $\sigma(\varepsilon)=c^{2} \varepsilon+D$ for $\varepsilon^{*}<\varepsilon<\max h(t)$, where $c$ and $D$ are constants. We take the density to be 1 so that $c$ is the sound speed.

(iv) The phase boundary is energy confining: acoustic waves impinging on the phase boundary are totally reflected, leaving unaltered the fields on the other side.

A more detailed explanation of how this problem can arise may be found in [5].

The trajectory $x=s(t)$ of the phase boundary is to be determined. Across this boundary the jump in the dynamical fields is restricted by the familiar shock conditions

$$
\frac{d s}{d t}[\varepsilon]+[v]=0, \quad \frac{d s}{d t}\lceil v]+[\sigma]=0,
$$

from which it follows that

$$
\dot{s}(0)=\left.(|\sigma| /|\varepsilon|)^{1 / 2}\right|_{t=0}=\left\{\left(\sigma^{*}-\sigma^{*}\right) /\left(\varepsilon^{*}-\varepsilon_{\mathrm{I}}\right)\right\}^{1 / 2}=0 .
$$

Thus, at least for short times, acoustic waves originating at the loading device overtake and reflect off the back of the phase boundary, driving this boundary forward into the region of constant strain. The ringing of these waves between the two boundaries of changing (unknown) separation accounts for the functional equation we now derive.

Let II be the as yet unknown region occuped by the material in the second phase. Thus

$$
\mathrm{II}=\{(x, t) \mid 0 \leqslant x<s(t), t>0\} .
$$

The governing equations in the interior of II are

$$
c^{2} \partial^{2} u / \partial x^{2}=\partial^{2} u / \partial t^{2}, \quad \sigma(\varepsilon)=c^{2} \varepsilon+D,
$$


with boundary condition $\varepsilon(0, t)=h(t)$ at $x=0$ and shock conditions (2.1) at $x=s(t)$. We assume, subject to later verification, that $s(t)$ is continuously differentiable and never characteristic. Thus $s(0)=\dot{s}(0)=0$ and $0<\dot{s}(t)<c$ for $t>0$. Under these conditions the displacement $u(x, t)$ for $(x, t) \in$ II can be written in terms of the data on $\xi=s(\eta)$ between the times $t_{a} \leqslant \eta \leqslant t_{b}$. Here $(\xi, \eta)$ are space and time coordinates. The times $t_{a}=t_{a}(x, t), t_{b}=t_{b}(x, t)$ delimit the domain of dependence on $\xi=s(\eta)$ for the point $(x, t)$ (Fig. 2). This data can be expressed in terms of the (constant) strain and velocity immediately ahead of the phase boundary via (2.1). One finds that

$u(x, t)=u(0,0)+\int_{0}^{t_{a}}\left[\dot{s}(\eta) \varepsilon_{\mathrm{I}}+v_{\mathrm{I}}\right] d \eta-\frac{1}{2 c} \int_{t_{a}}^{t_{b}}\left\{[\dot{s}(\eta)-c] v_{\mathrm{I}}-c \dot{s}(\eta) \varepsilon_{\mathrm{I}}+c^{2} \varepsilon^{*}\right\} d \eta$.

The trajectory $s(\eta)$ must be chosen so as to satisfy the unused condition $\varepsilon(0, t)=h(t)$. The strain $\varepsilon(0, t)$ can be calculated from (2.2) and expressed in terms of $\dot{a}(t)$ and $\dot{b}(t)$ where $a(t) \equiv t_{a}(0, t), b(t) \equiv t_{b}(0, t)$. The result is

$$
h(t)=\frac{1}{2}\left(\varepsilon^{*}-\varepsilon_{\mathrm{I}}\right)(\dot{b}(t)+\dot{a}(t))+\varepsilon_{\mathrm{I}} .
$$

The missing steps in the derivation of (2.2) and (2.3) are given in the appendix, where we treat a more general case in which (ii) need not hold.

By virtue of $a(0)=0, b(0)=0$, Eq. (2.3) integrates to

$$
a(t)+b(t)=2 t+\frac{1}{2} Q(t)
$$

where

$$
Q(t)=\frac{4}{\left(\varepsilon^{*}-\varepsilon_{\mathrm{I}}\right)} \int_{0}^{t}\left[h(r)-\varepsilon^{*}\right] d r .
$$

We note for future reference that $Q$ is of unlimited smoothness with

$$
Q(0)=\dot{Q}(0)=0, \quad \dot{Q}(z)>0 \quad(z>0) .
$$

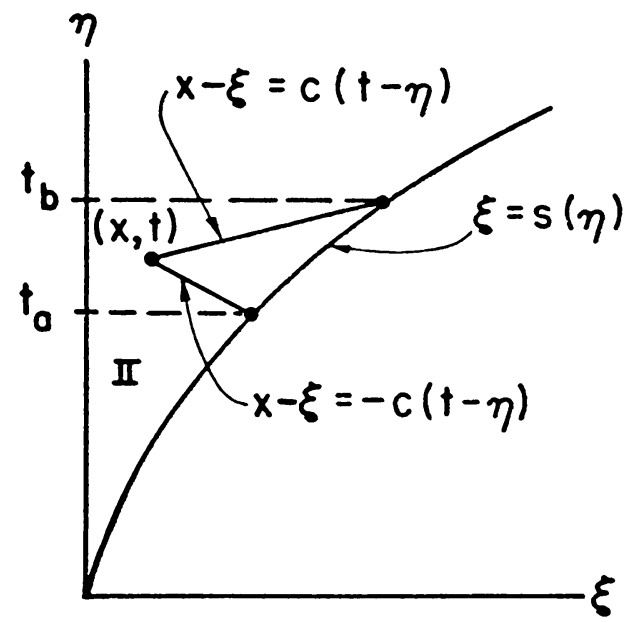

FIG. 2 
The functions $a(t)$ and $b(t)$ are connected to $s(t)$ by the implicit relations:

$$
\begin{gathered}
s(a(t))-c[t-a(t)]=0, \\
s(b(t))+c[t-b(t)]=0, \\
a(0)=b(0)=0 .
\end{gathered}
$$

The assumption $0<\dot{s}(t)<c$ for $t>0$ ensures that $a(t)$ and $b(t)$ are each increasing. Let $a^{-1}(\cdot)$ and $b^{-1}(\cdot)$ be their respective inverse functions. Eliminating $s(\cdot)$ from the above relations yields

$$
a^{-1}(t)+b^{-1}(t)=2 t
$$

We remark that $a^{-1}(b(t))-t$ is the elapsed time for the acoustic wave generated at time $t$ to reflect off the phase boundary and return to $x=0$. Our final form is found by introducing $\varphi(z)=2 a(z)-z$. Then (2.6) requires that

$$
b(\varphi(z))=\frac{1}{2} \varphi(z)+\frac{1}{2} z,
$$

whereas (2.4) demands that

$$
b(\varphi(z))=\frac{1}{2} Q(\varphi(z))-\frac{1}{2} \varphi(\varphi(z))+\frac{3}{2} \varphi(z) .
$$

Together these give

$$
\varphi(\varphi(z))-2 \varphi(z)+z=Q(\varphi(z)), \quad z \geqslant 0 .
$$

The phase boundary $s(t)$ is given in terms of $\varphi$ as

$$
s(t)=c\left[a^{-1}(t)-t\right], \quad a(t)=\frac{1}{2}[\varphi(t)+t] .
$$

The assumptions on $s(t)$ are equivalent to $\varphi$ being continuously differentiable with

$$
\varphi(0)=0, \quad \dot{\varphi}(0)=1, \quad 0<\dot{\varphi}(z)<1 \quad \text { for } z>0 .
$$

In general we cannot expect higher derivatives of $\varphi$ to exist at $z=0$ without additional assumptions on $h(t)$. To show this we suppose that

$$
Q(z)=\frac{1}{2} \ddot{Q}(0) z^{2}+O\left(z^{3}\right),
$$

and by virtue of (2.9) attempt a small $t$ expansion for $\varphi$ of the form

$$
\varphi(t)=t+a t^{k}+o\left(t^{k}\right), \quad k>1 .
$$

Then

$$
\varphi(\varphi(t))-2 \varphi(t)+t=k a^{2} t^{2 k-1}+o\left(t^{2 k-1}\right)
$$

and

$$
Q(\varphi(t))=\frac{1}{2} \ddot{Q}(0) t^{2}+o\left(t^{2}\right)
$$

This implies

$$
\varphi(t)=t-(\ddot{Q}(0) / 3)^{1 / 2} t^{3 / 2}+o\left(t^{3 / 2}\right),
$$

which in turn yields

$$
s(t)=\frac{1}{2} c(\ddot{Q}(0) / 3)^{1 / 2} t^{3 / 2}+o\left(t^{3 / 2}\right)=c\left(\frac{1}{3} \dot{h}(0+) /\left(\varepsilon^{*}-\varepsilon_{\mathrm{I}}\right)\right)^{1 / 2} t^{3 / 2}+o\left(t^{3 / 2}\right) .
$$


3. Existence of a solution to the functional equation. In this section, we prove that (2.5), (2.7) has a unique solution obeying (2.9). In addition, the first of the two theorems in this section furnishes an iterative scheme for solving (2.7).

Let $R^{+}=\{x \mid x \geqslant 0\}$, and for $n=0,1,2, \ldots$ let

$$
\varphi^{[n]}(x)=\varphi(\varphi(\varphi(\cdots(\varphi(x)) \cdots))), \quad(n \text {-times }) \text { and } \varphi^{[0]}(x)=x .
$$

Define the set of functions

$$
\begin{gathered}
\mathscr{S}=\left\{\varphi: R^{+} \rightarrow R^{+} \mid \varphi(0)=0, \quad \varphi(t)\right. \text { strictly increasing, } \\
\text { and } \varphi \operatorname{Lipschitz~with~} \operatorname{Lip}[\varphi] \leqslant 1\} .
\end{gathered}
$$

Note that $\mathscr{S}$ is closed under composition. The following theorem shows that (2.7) has a unique solution $\varphi \in \mathscr{S}$ for a class of functions $Q$ which include those $Q$ obeying (2.5).

THEOREM 1. Let $Q: R^{+} \rightarrow R^{+}$be continuous and strictly increasing with $Q(0)=0$. Then the equation

$$
\varphi(\varphi(t))-2 \varphi(t)+t-Q(\varphi(t))=0 \quad(t \geqslant 0),
$$

possesses a solution $\varphi \in \mathscr{S}$. Moreover this solution $\varphi$ obeys

$$
0<\varphi(t)<t \quad(t>0),
$$

and is the only solution of (3.2) in $\mathscr{S}$.

Proof. Define $L: \mathscr{S} \rightarrow \mathscr{S}$ by

$$
L \psi(t)=\frac{1}{2} \psi(\psi(t))+\frac{1}{2} t .
$$

Also define $K: R^{+} \rightarrow R^{+}$by $K(z)=z+\frac{1}{2} Q(z)$. The hypotheses on $Q$ ensure that $K$ is invertible on $R^{+}$with the inverse function $K^{-1}$ obeying

$$
0<K^{-1}(z)<z \quad(z>0) .
$$

Furthermore, one may verify that $K^{-1} \in \mathscr{S}$.

We may write (3.2) either as

$$
K(\varphi(t))=L \varphi(t) \quad(t \geqslant 0),
$$

or as

$$
\varphi(t)=K^{-1}(L \varphi(t)) \quad(t \geqslant 0) .
$$

We are first going to show that the sequence of functions

$$
\varphi_{n}(t)=K^{-1}\left(L \varphi_{n-1}(t)\right), \quad \varphi_{1}(t)=K^{-1}\left(\frac{1}{2} t\right),
$$

converges pointwise to a solution $\varphi \in \mathscr{S}$ of (3.5).

We have $0 \leqslant \varphi_{n}(t) \leqslant t$ since $\varphi_{n} \in \mathscr{S}$. An inductive argument now gives $\varphi_{n}(t) \geqslant \varphi_{n-1}(t)$; thus $\varphi_{n}(t) \rightarrow \varphi(t)$ for all $t \geqslant 0$. Since $\varphi_{n} \in \mathscr{S}$, it follows that $\varphi(t)$ is (i) Lipschitz with $\operatorname{Lip}[\varphi] \leqslant 1$, (ii) increasing, and (iii) obeys $\varphi(0)=0$. Since $L \varphi_{n}(t) \rightarrow L \varphi(t)$ and $K \varphi_{n}(t)$ $\rightarrow K \varphi(t)$ it is immediate from (3.7) that $\varphi$ is a solution of (3.5) or, equivalently, (3.2). From (3.2) we have $t_{1} \neq t_{2}$ implies $\varphi\left(t_{1}\right) \neq \varphi\left(t_{2}\right)$, whence $\varphi$ is strictly increasing and so $\varphi \in \mathscr{S}$. In addition, for $t>0$ we have $\varphi(t)>0$, so that $K^{-1}(L \varphi(t))<L \varphi(t) \leqslant t$ by (3.4). Since $K^{-1}(L \varphi(t))=\varphi(t)$, this establishes (3.3). It remains to show that $\varphi$ is the unique solution of (3.2) in $\mathscr{S}$. 
It is immediate from (3.3) that

$$
\lim _{n \rightarrow \infty} \varphi^{[n]}(t)=0 \quad(t>0) .
$$

Suppose now that $\psi \in \mathscr{S}$ is also a solution of (3.2). Then

$$
\begin{aligned}
|\varphi(t)-\psi(t)| & =\left|K^{-1}(L \varphi(t))-K^{-1}(L \psi(t))\right| \\
& \leqslant|L \varphi(t)-L \psi(t)|=\frac{1}{2}|\varphi(\varphi(t))-\psi(\psi(t))| \\
& \leqslant \frac{1}{2}|\varphi(\varphi(t))-\psi(\varphi(t))|+\frac{1}{2}|\psi(\varphi(t))-\psi(\psi(t))| \\
& \leqslant \frac{1}{2}|\varphi(\varphi(t)-\psi(\varphi(t)))|+\frac{1}{2}|\varphi(t)-\psi(t)|,
\end{aligned}
$$

whence

$$
|\varphi(t)-\psi(t)| \leqslant|\varphi(\varphi(t))-\psi(\varphi(t))| .
$$

This gives, by iteration,

$$
|\varphi(t)-\psi(t)| \leqslant\left|\varphi\left(\varphi^{[n]}(t)\right)-\psi\left(\varphi^{[n]}(t)\right)\right|,
$$

for all $n=1,2,3 \ldots$ and all $t \geqslant 0$. Taking the limit of (3.11) as $n \rightarrow \infty$ and using (3.8) we arrive at

$$
|\varphi(t)-\psi(t)| \leqslant|\varphi(0)-\psi(0)|=0,
$$

hence $\psi=\varphi$.

Theorem 1 implies that $\dot{\varphi}$ exists a.e. on $R^{+}$with $0 \leqslant \dot{\varphi} \leqslant 1$ and moreover that $\varphi(t)=\int_{0}^{t} \dot{\varphi}(s) d s$, the integral being in the sense of Lebesgue. Our next aim is to show that $\varphi$ is continuously differentiable and satisfies (2.9). Let $D \subset R^{+}$be a Lebesgue measurable set with $\mu(D)=0$ such that $\dot{\varphi}$ exists for all $t \in D^{\mathrm{c}}$. Here and throughout the section, $\mu$ will denote Lebesgue measure and ${ }^{c}$ will denote set complement in $R^{+}$. Differentiating (3.2) gives

$$
(\dot{\varphi}(\varphi(t))-2-\dot{Q}(\varphi(t))) \dot{\varphi}(t)+1=0,
$$

for all $t \in D^{\mathrm{c}}$ such that $\varphi(t) \in D^{\mathrm{c}}$. Since $t \in D^{\mathrm{c}}$ need not imply $\varphi(t) \in D^{\mathrm{c}}$, it is inconvenient to work with the sets $D$ and $D^{\mathrm{c}}$. Instead, we seek a set $F \subset R^{+}$such that (i) $\mu(F)=0$, (ii) $\dot{\varphi}$ exists for all $t \in F^{\mathrm{c}}$, and (iii) $\varphi\left(F^{\mathrm{c}}\right) \subset F^{\mathrm{c}}$. Clearly the set

$$
F=\bigcup_{n=0}^{\infty}\left\{\left(\varphi^{-1}\right)^{[n]}(D)\right\}
$$

satisfies the latter two requirements. Thus (3.13) holds for all $t \in F^{\mathrm{c}}$. To show that $\mu(F)=0$ we note that $\varphi \in \mathscr{S}$ ensures that $\varphi^{-1}$ is a strictly increasing function. Moreover, (3.2) gives explicitly

$$
\varphi^{-1}(t)=2 t+Q(t)-\varphi(t) .
$$

Suppose $Q$ is absolutely continuous. Then so is $\varphi^{-1}$ which, in turn, renders $\mu(F)=0$. In particular, $Q$ is absolutely continuous under the hypotheses of:

THeOREM 2. Let $Q$ be continuously differentiable with $Q(0)=\dot{Q}(0)=0, \dot{Q}(z)>0$ for $z>0$ and, in addition, let $\dot{Q}$ be Lipschitz on every interval $\left[0, z_{0}\right]$. Then the solution $\varphi \in \mathscr{S}$ of (3.2) is continuously differentiable with $\dot{\varphi}(0)=1$ and $0<\dot{\varphi}(t)<1$ for all $t>0$. 
The theorem will be proved in several stages. The hypotheses of Theorem 2 are assumed throughout.

Lemma 1. Let $A: B \rightarrow R^{+}$, where the set $B \subset R^{+}$is such that $\varphi(B) \subset B$. Let $A$ obey

$$
0 \leqslant A(t) \leqslant 1 \text {, }
$$

and

$$
[A(\varphi(t))-2-\dot{Q}(\varphi(t))] A(t)+1=0,
$$

for all $t \in B$. If $t_{k} \in B$ and $t_{k} \rightarrow 0$, then $A\left(t_{k}\right) \rightarrow 1$.

Proof. If $A\left(t_{k}\right) \nrightarrow 1$, we conclude from (3.16) the existence of a subsequence $t_{k}$, such that $A\left(t_{k_{t}}\right) \rightarrow \gamma_{0}, 0 \leqslant \gamma_{0}<1$. Thus it is sufficient to show that if $A\left(t_{k}\right) \rightarrow \gamma_{0}$, then $\gamma_{0}=1$.

For each $n=0,1,2, \ldots$, (3.3) yields

$$
\lim _{k \rightarrow \infty} \varphi^{[n]}\left(t_{k}\right)=0, \quad \lim _{k \rightarrow \infty} \dot{Q}\left(\varphi^{[n]}\left(t_{k}\right)\right)=0 .
$$

Since $\varphi(B) \subset B,(3.17)$ ensures the existence of each limit

$$
\gamma_{n}=\lim _{k \rightarrow \infty} A\left(\varphi^{[n]}\left(t_{k}\right)\right), \quad n=1,2, \ldots,
$$

obeying

$$
\gamma_{n}=2-1 / \gamma_{n-1}, \quad n=1,2, \ldots,
$$

which is the same as

$$
1-\gamma_{n}=\left(1-\gamma_{n-1}\right) / \gamma_{n-1}, \quad n=1,2, \ldots
$$

From (3.19), (3.16) and the definition of $\gamma_{0}$, we have $0 \leqslant \gamma_{n} \leqslant 1(n=0,1, \ldots)$. Thus (3.21) gives $\gamma_{n} \leqslant \gamma_{n-1}$. Hence $\gamma_{n} \rightarrow \gamma$ with $1-\gamma=(1-\gamma) / \gamma$, so $\gamma=1$. Collecting results we have $1=\gamma=\lim _{n \rightarrow \infty} \gamma_{n} \leqslant \gamma_{0} \leqslant 1$, whence $\gamma_{0}=1$.

Two sets which satisfy the requirements upon $B$ stated above are $F^{\mathrm{c}}$ and $R^{+}$. Note that $\dot{\varphi}$ obeys (3.16), (3.17) for $B=F^{c}$. Thus we draw three corollaries central to the following development.

Corollary 1. All $A: F^{\mathrm{c}} \rightarrow R^{+}$obeying (3.16), (3.17) on $F^{\mathrm{c}}$ have the property that $A\left(t_{k}\right) \rightarrow 1$ whenever $t_{k} \rightarrow 0, t_{k} \in F^{\mathrm{c}}$.

CorollaRY 2. $\dot{\varphi}\left(t_{k}\right) \rightarrow 1$ for all $t_{k} \rightarrow 0, t_{k} \in F^{c}$.

Corollary 3. If $A: R^{+} \rightarrow R^{+}$obeys (3.16), (3.17) on $R^{+}$, then $A$ is continuous at the origin with $A(0)=1$.

The first two corollaries guarantee that $\dot{\varphi}$ is the unique solution of (3.17) in the following sense.

Lemma 2. If $A: R^{+} \rightarrow R^{+}$obeys (3.16), (3.17) for all $t \in F^{\mathrm{c}}$, then $A(t)=\dot{\varphi}(t)$ for all $t \in F^{\mathrm{c}}$. 
Proof. Write (3.17) in the form

$$
A(t)=-1 /[A(\varphi(t))-2-\dot{Q}(\varphi(t))] ;
$$

a corresponding result holds with $\dot{\varphi}$ replacing $A$. By subtracting these two expressions and invoking (3.16), $0 \leqslant \dot{\varphi} \leqslant 1$, and $\dot{Q}(\varphi(t)) \geqslant 0$, we arrive at

$$
|A(t)-\dot{\varphi}(t)| \leqslant|A(\dot{\varphi}(t))-\dot{\varphi}(\varphi(t))| \quad\left(t \in F^{\mathrm{c}}\right),
$$

which gives, by induction since $\varphi\left(F^{\mathrm{c}}\right) \subset F^{\mathrm{c}}$,

$$
|A(t)-\dot{\varphi}(t)| \leqslant\left|A\left(\varphi^{[n]}(t)\right)-\dot{\varphi}\left(\varphi^{[n]}(t)\right)\right| \quad\left(t \in F^{\mathrm{c}}, n=1,2, \ldots\right) .
$$

Letting $n \rightarrow \infty$ we have from (3.8) and Corollaries 1 and 2,

$$
|A(t)-\dot{\varphi}(t)| \leqslant \lim _{\substack{r \rightarrow 0 \\ r \in F^{\mathrm{c}}}}|A(r)-\dot{\varphi}(r)|=0 \quad\left(t \in F^{\mathrm{c}}\right) .
$$

We already have that $\dot{\varphi}$ is a solution of (3.17) on $F^{\mathrm{c}}$. We now show that (3.17) possesses a solution not only on $F^{\mathrm{c}}$, but also on $R^{+}$.

Lemma 3. There exists $A: R^{+} \rightarrow R^{+}$, continuous with $A(0)=1,0<A(t)<1$ for $t>0$ such that (3.17) holds for all $t \in R^{+}$.

Proof. Consider the sequence of functions generated by

$$
A_{k+1}(t)=1 /\left\{2+\dot{Q}(\varphi(t))-A_{k}(\varphi(t))\right\}, \quad A_{1}(T)=1 /\{2+\dot{Q}(\varphi(t))\} .
$$

Separate arguments based upon induction and $\dot{Q}(z) \geqslant 0$ yield $0<A_{k}(t) \leqslant 1(t \geqslant 0)$, and $A_{k+1}(t) \geqslant A_{k}(t)(t \geqslant 0)$. Hence $A(t) \equiv \lim _{k \rightarrow \infty} A_{k}(t)$ exists and obeys $0 \leqslant A(t) \leqslant 1$. It is evident from (3.25) that $A(t)$ is a solution of (3.17). Also

$$
A(0)=1 /\{2+\dot{Q}(\varphi(0))-A(\varphi(0))\}=1 /\{2-A(0)\}
$$

so that $A(0)=1$. Moreover, for $t>0$, the strict inequality $Q(\varphi(t))>0$ furnishes

$$
1 / A(t)=2+\dot{Q}(\varphi(t))-A(\varphi(t))>2-A(\varphi(t)) \geqslant 1,
$$

whence $0<A(t)<1$ for $t>0$. The continuity of $A$ at the origin is the result of Corollary 3. It remains to show that $A(t)$ is continuous for $t>0$.

Let $t>0$ and $\varepsilon>0$ be given; we shall show that there exists $\delta>0$ such that if $\tau>0$ with $|t-\tau|<\delta$ then $|A(t)-A(\tau)|<\varepsilon$. Let $q$ be a Lipschitz constant for $\dot{Q}$ on $0 \leqslant z \leqslant t+1$. Choose $d>0$ such that $0 \leqslant x \leqslant d, 0 \leqslant y \leqslant d$ implies $|A(x)-A(y)|<$ $\varepsilon / 2$. Now (3.8) guarantees the existence of an $N$ such that $\varphi^{[N]}(t+1)<d$. From (3.17) and the bounds $A(z) \leqslant 1, \dot{Q}(z) \geqslant 0$, it follows that

$$
|A(t)-A(\tau)| \leqslant|\dot{Q}(\varphi(\tau))-\dot{Q}(\varphi(t))|+|A(\varphi(t))-A(\varphi(\tau))| \quad(t \geqslant 0, \tau \geqslant 0) .
$$

Iterating this inequality $N-1$ times, we arrive at

$$
|A(t)-A(\tau)| \leqslant\left\{\sum_{k=1}^{N}\left|\dot{Q}\left(\varphi^{[k]}(t)\right)-\dot{Q}\left(\varphi^{[k]}(\tau)\right)\right|\right\}+\left|A\left(\varphi^{[N]}(t)\right)-A\left(\varphi^{[N]}(\tau)\right)\right| .
$$


Let $\delta=\min \{1, \varepsilon /(2 N q)\}$ and suppose that $|t-\tau|<\delta$. Then (3.27) implies

$$
|A(t)-A(\tau)|<\left\{\sum_{k=1}^{N} q|t-\tau|\right\}+\frac{\varepsilon}{2} \leqslant \varepsilon .
$$

We now rapidly complete the

Proof of Theorem 2. Let $A$ be as given in Lemma 3. Then, since $\mu(F)=0$, Lemmas 2 and 3 furnish

$$
\varphi(t)=\int_{0}^{t} \dot{\varphi}(s) d s=\int_{0}^{t} A(s) d s .
$$

In view of the continuity of $A$, the latter integral is a Riemann integral. Hence, by the Fundamental Theorem of Integral Calculus, $\dot{\varphi}(t)$ exists for all $t>0$ and $\dot{\varphi}(t)=A(t)$. Finally, Lemma 3 ensures all the requisite properties of $\dot{\varphi}$.

4. Remarks. In Sec. 2 we touched upon the short-time behavior of solutions. The long-time behavior can also be addressed. If we suppose that $h(t) \rightarrow \varepsilon_{\infty}>\varepsilon^{*}$ then

$$
Q(\varphi(t)) \sim 4\left(\left(\varepsilon_{\infty}-\varepsilon^{*}\right) /\left(\varepsilon^{*}-\varepsilon_{\mathrm{I}}\right)\right) \varphi(t)
$$

and (2.7) yields $\varphi(t) \sim \gamma t$ where $\gamma$ is the root of a quadratic equation. This root is uniquely determined from the requirement that $0<\gamma<1$ and leads to

$$
s(t) \sim\left(\left(\varepsilon_{\infty}-\varepsilon^{*}\right) /\left(\varepsilon_{\infty}-\varepsilon_{1}\right)\right)^{1 / 2} c t .
$$

This indicates that the square of the asymptotic phase boundary speed is given by the slope of the secant line on the $\sigma(\varepsilon)$-curve connecting $\left(\varepsilon_{\mathrm{I}}, \sigma^{*}\right)$ to $\left(\varepsilon_{x}, \sigma\left(\varepsilon_{\infty}\right)\right)$.

Equations where a function, which is to be determined, appears in the argument of another function, arise in other wave propagation problems involving an unknown time delay for the return of a reflected signal. Mortell and Seymour [11] consider a set of functional equations in connection with the study of oscillations occurring in an inviscid gas confined in a closed tube. In fact, their equation (1) can be manipulated into the form of (2.7), although the properties of $Q$ ( $H$ in [11]) differ. The reason for the signal delay is also different; in [11] the location of the reflecting barriers are known whereas the sound speed-being amplitude dependent-must be determined. A different set of functional equations arise in the one-dimensional theory of elastic-plastic unloading waves. By showing that a complete solution follows from the solution on the initial time interval, Skobeev [9] gives an existence theorem for this set. The difficulty lies in analyzing a sequence of problems with successively shorter initial intervals on which it is possible to find the solution. In contrast, the iterative method (3.7) for our problem does not require the analysis of $\varphi(t)$ on an initial interval.

The success of the procedure used here is connected to the simple form of the final equation (2.7). A more complicated formulation results if we drop assumption (ii) of Sec. 2 and study a phase boundary advancing into a more general strain and velocity field. In this case (2.6) holds but (2.4) does not. The latter gives way to a modified form of (2.3) which is derived in the appendix. Then it is found that $h(t)$ remains linear in $\dot{a}(t)$ and $\dot{b}(t)$, but the coefficients are complicated functions which involve the unknowns $a(t)$ and $b(t)$ in their arguments. 
Acknowledgment. The author would like to acknowledge helpful discussions with R. D. James, W. Hrusa, R. Lyons, M. Renardy, and R. E. L. Turner.

Appendix. A phase boundary advancing into a general strain and velocity field. Consider a phase boundary $\xi=s(\eta)$ obeying (i), (iii), and (iv) of Sec. 2. Integrating the equation $c^{2} \partial^{2} u / \partial x^{2}-\partial^{2} u / \partial t^{2}=0$ over the triangular region of Fig. 2 and applying Green's theorem yields the analogue of d'Alembert's formula:

$$
\begin{aligned}
u(x, t)= & \frac{1}{2}\left\{u\left(s\left(t_{a}\right), t_{a}\right)+u\left(s\left(t_{b}\right), t_{b}\right)\right\} \\
& -\frac{1}{2 c} \int_{t_{a}}^{t_{b}}\left\{v(s(\eta)-, \eta) \dot{s}(\eta)+c^{2} \varepsilon(s(\eta)-, \eta)\right\} d \eta .
\end{aligned}
$$

Here the argument $(\xi, \eta)=(s(\eta)-, \eta)$ refers to a coordinate immediately behind the phase boundary. It is desired to express the quantities appearing in (A.1) in terms of $\varepsilon(s(\eta)+, \eta)$ and $v(s(\eta)+, \eta)$. Now

$$
\begin{aligned}
u(s(\tau), \tau) & =u(0,0)+\int_{0}^{\tau}\{\varepsilon(s(\eta)-, \eta) \dot{s}(\eta)+v(s(\eta)-, \eta)\} d \eta \\
& =u(0,0)+\int_{0}^{\tau}\{\varepsilon(s(\eta)+, \eta) \dot{s}(\eta)+v(s(\eta)+, \eta)\} d \eta,
\end{aligned}
$$

since $u(x, t)$ is continuous across $\xi=s(\eta)$ (see $\left.(2.1)_{1}\right)$. The shock conditions (2.1) also furnish

$v(s(\eta)-, \eta) \dot{s}(\eta)+c^{2} \varepsilon(s(\eta)-, \eta)=v(s(\eta)+, \eta) \dot{s}(\eta)+\sigma(\varepsilon(s(\eta)+, \eta))-D$.

Substituting from (A.2), (A.3) into (A.1) gives

$$
\begin{aligned}
& u(x, t)=u(0,0)+\int_{0}^{t_{a}}\{\dot{s}(\eta) \varepsilon(s(\eta)+, \eta)+v(s(\eta)+, \eta)\} d \eta \\
& -\frac{1}{2 c} \int_{t_{a}}^{t_{b}}\{(\dot{s}(\eta)-c) v(s(\eta)+, \eta)-c \dot{s}(\eta) \varepsilon(s(\eta)+, \eta)+\sigma(\varepsilon(s(\eta)+, \eta))-D\} d \eta .
\end{aligned}
$$

To obtain an expression for $\varepsilon(0, t)$ we differentiate (A.4) with respect to $x$ and then set $x=0$. This gives

$$
\begin{aligned}
& h(t)=\varepsilon(0, t) \\
& \quad=\left.\frac{1}{2 c} \frac{\partial t_{a}}{\partial x}\right|_{(0, t)} \times\left.\{c \dot{s}(\eta) \varepsilon(\xi, \eta)+(\dot{s}(\eta)+c) v(\xi, \eta)+\sigma(\varepsilon(\xi, \eta))-D\}\right|_{(\xi, \eta)=(s(a(t))+, a(t))} \\
& -\left.\frac{1}{2 c} \frac{\partial t_{b}}{\partial x}\right|_{(0, t)} \times\left.\{(\dot{s}(\eta)-c) v(\xi, \eta)-c \dot{s}(\eta) \varepsilon(\xi, \eta)+\sigma(\varepsilon(\xi, \eta))-D\}\right|_{(\xi, \eta)=(s(b(t))+, b(t))}
\end{aligned}
$$

The functions $t_{a}=t_{a}(x, t)$ and $t_{b}=t_{b}(x, t)$ are defined implicitly in terms of the trajectory $s(\cdot)$ as the roots of

$$
x=s\left(t_{b}\right)+c\left(t-t_{b}\right), \quad x=s\left(t_{a}\right)-c\left(t-t_{a}\right),
$$

and $a(t) \equiv t_{a}(0, t), b(t) \equiv t_{b}(0, t)$. Thus

$$
\dot{s}(a(t))=\frac{c}{\dot{a}(t)}-c, \quad \dot{s}(b(t))=-\frac{c}{\dot{b}(t)}+c,
$$


and

$$
\begin{aligned}
& \left.\frac{\partial t_{a}}{\partial x}\right|_{(0, t)}=\frac{1}{\dot{s}\left(t_{a}(0, t)\right)+c}=\frac{\dot{a}(t)}{c}, \\
& \left.\frac{\partial t_{b}}{\partial x}\right|_{(0, t)}=\frac{1}{\dot{s}\left(t_{b}(0, t)\right)-c}=-\frac{\dot{b}(t)}{c} .
\end{aligned}
$$

In view of (A.7), (A.8) the equation (A.5) becomes

$$
\begin{aligned}
\dot{h}(t)= & \left.\frac{\dot{a}(t)}{2 c^{2}}\left\{\sigma(\varepsilon(\xi, \eta))-D-c^{2} \varepsilon(\xi, \eta)\right\}\right|_{(\xi, \eta)=(s(a(t))+, a(t))} \\
& +\left.\frac{\dot{b}(t)}{2 c^{2}}\left\{\sigma(\varepsilon(\xi, \eta))-D-c^{2} \varepsilon(\xi, \eta)\right\}\right|_{(\xi, \eta)=(s(b(t))+, b(t))} \\
& +\left.\frac{1}{2 c^{2}}\left\{c^{2} \varepsilon(\xi, \eta)+c v(\xi, \eta)\right\}\right|_{(\xi, \eta)=(s(a(t))+, a(t))} \\
& +\left.\frac{1}{2 c^{2}}\left\{c^{2} \varepsilon(\xi, \eta)-c v(\xi, \eta)\right\}\right|_{(\xi, \eta)=(s(b(t))+, b(t))} .
\end{aligned}
$$

For the case in which (ii) of Sec. 2 also holds, one has

$$
\begin{gathered}
\varepsilon(s(\eta)+, \eta)=\varepsilon_{\mathrm{I}}, \\
v(s(\eta)+, \eta)=v_{\mathrm{I}}, \\
\sigma(\varepsilon(s(\eta)+, \eta))-D=c^{2} \varepsilon^{*}
\end{gathered}
$$

so that (A.4), (A.9) reduce to $(2.2),(2.3)$.

\section{REFERENCES}

[1] D. E. Grady, R. E. Hollenbach, and K. W. Schuler, Compression wave studies in calcite rock, J. Geophys. Res. 83, 2839-2849 (1978)

[2] J. L. Ericksen, Equilibrium of bars, J. Elasticity 5, 191-201 (1975)

[3] R. D. James, Co-existent phases in the one-dimensional static theory of elastic bars, Arch. Rat. Mech. Anal. 72, 99-140 (1979)

[4] R. D. James, The propagation of phase boundaries in elastic bars, Arch. Rat. Mech. Anal. 73, 125-150 (1980)

[5] T. J. Pence, On the emergence and propagation of a phase boundary in an elastic bar with a suddenly applied end load, J. Elasticity 16, 3-42 (1986)

[6] J. Serrin, Phase transitions and interfacial layers for van der Waals fluids, in Proceedings of SAFA IV Conference, Recent Methods in Nonlinear Analysis and Applications, Naples, March 21-28, 1980 (A. Canfora, S. Rionero, C. Sbordone, C. Trombetti, Eds.)

[7] M. Slemrod, Admissibility criteria for propagating phase boundaries in a van der Waals fluid, Arch. Rat. Mech. Anal. 81, 301-315 (1983)

[8] R. Hagan and M. Slemrod, The viscosity-capillarity criterion for shocks and phase transitions, Arch. Rat. Mech. Anal. 83, 333-361 (1983)

[9] A. M. Skobeev, On the theory of unloading waves, Appl. Math. Mech., PMM, 26, 1605-1615 (1963)

[10] R. J. Clifton and S. R. Bodner, An analysis of longitudinal elastic-plastic pulse propagation, J. Appl. Mech. 33, 248-255 (1966)

[11] M. P. Mortell and B. R. Seymour, Exact solutions of a functional equation arising in nonlinear wave propagation, SIAM J. Appl. Math. 30, 587-596 (1976) 